\title{
Regulation of D-galacturonate metabolism in Caulobacter crescentus by HumR, a LacI-family transcriptional repressor
}

\author{
Aaesha I. Sheikh, Deborah Caswell, Cynthia Dick, Spencer Gang, Justin Jarrell, Ankita Kohli, \\ Amanda Lieu, Jared Lumpe, Meghan Garrett, Jennifer Parker, Craig Stephens \\ Biology Department, Santa Clara University, 500 El Camino Real, Santa Clara, USA \\ Email: Cstephens@scu.edu
}

Received 28 July 2013; revised 28 August 2013; accepted 19 September 2013

Copyright (C) 2013 Aaesha I. Sheikh et al. This is an open access article distributed under the Creative Commons Attribution License, which permits unrestricted use, distribution, and reproduction in any medium, provided the original work is properly cited.

\begin{abstract}
The oligotrophic freshwater bacterium Caulobacter crescentus encodes a cluster of genes (CC_1487 to CC_1495) shown here to be necessary for metabolism of D-galacturonate, the primary constituent of pectin, a major plant polymer. Sequence analysis suggests that these genes encode a version of the bacterial hexuronate isomerase pathway. A conserved 14 bp sequence motif is associated with promoter regions of three operons within this cluster, and is conserved in homologous gene clusters in related alpha-Proteobacteria. Embedded in the hexuronate gene cluster is a gene (CC_1489) encoding a member of the LacI family of bacterial transcription factors. This gene product, designated here as HumR (hexuronate metabolism regulator), represses expression of the $u x a A$ and uxaC operon promoters by binding to the conserved operator sequence. Repression is relieved in the presence of galacturonate or, to a lesser extent, by glucuronate. Other genes potentially involved in pectin degradation and hexuronate transport are also under the control of HumR. Adoption of a LacI-type repressor to control hexuronate metabolism parallels the regulation of xylose, glucose, and maltose utilization in C. crescentus, but is distinct from the use of GntRtype repressors to control pectin and hexuronate utilization in gamma-Proteobacteria such as Escherichia coli.
\end{abstract}

Keywords: Caulobacter; Galacturonate; LacI

\section{INTRODUCTION}

Pectin is an important plant polysaccharide that aids the cross-linking of cellulose and hemicellulose to maintain the integrity of plant cells and tissues [1]. The core pectin polymer consists of alpha-1, 4-linked D-galacturonate subunits, which are then modified by methylation and various oligosaccharide side chains depending on the plant source [1]. Metabolism of galacturonate, which makes up $40 \%-70 \%$ of the dry weight of pectin, has been studied extensively in just a few microbes [2,3]. In this work, we show that the oligotrophic, stalked alpha-Proteobacterium Caulobacter crescentus degrades galacturonate through the hexuronateisomerase pathway, and regulates galacturonate metabolism via LacI-family repressor. This system for galacturonate metabolism and regulation is conserved in several closely related alpha-Proteobacteria, although the metabolic pathway had previously been observed only in gamma-Proteobacteria such as Escherichia coli and Erwinia chrysanthemi.

Microorganisms degrade pectin through a suite of secreted enzymes, with pectatelyase and exo-polygalacturonidase primarily responsible for chain depolymerization $[2,4]$. The most extensively studied pectinolytic bacterium is the phytopathogen Erwinia chrysanthemi, which attacks pectin to facilitate invasion of plant tissue during infection [5]. Erwinia extracts nutrients from pectin degradation products by consuming oligo-galacturonides, galacturonate, and 5-keto-4-deoxyuronate through the hexuronatei somerase pathway it shares with non-phytopathogenic Enterobacteriaceae such as E. coli [3].

Caulobacters are dimorphic alpha-Proteobacteria that often exhibit an adhesive stalk and readily form biofilms [6]. It is likely that species such as C. crescentus colonize decaying vegetation in aquatic habitats, secreting hydrolytic enzymes to access nutrients embedded in the insoluble cell walls [7]. Whole genome sequencing of $C$. crescentus revealed numerous genes encoding secretable enzymes for attacking cellulose, xylans, pectin, and other polymers [8]. Genomic analysis of closely related stalked 
bacteria such as Caulobacter henriciistrain K31 [9] and Phenylobacterium zucineum [10] shows comparable rosters of secretable hydrolases. In $C$. crescentus, there is evidence for coordinated expression of gene sets that include extracellular enzymes, transport systems, and cytoplasmic catabolic pathways when cells are exposed to relevant sugars, such as glucose and xylose [11]. For example, transcriptome analysis of $C$. crescentus metabolizing xylose revealed induction of xylanases and arabinosidases, putative outer and inner-membrane transporters, and a pathway for oxidative metabolism of xylose $[11,12]$. The xylose regulon is under the control of XylR, a member of the LacI family of bacterial transcription factors [13]. Our results reveal an analogous galacturonate regulon in $C$. crescentus that is under the control of another LacI family member, HumR. Given that the xylose operon promoter [14], in conjunction with XylR [13], has proven to be a robust tool for tightly controlled inducible gene expression in C. crescentus, the identification of HumR not only reveals commonalities in regulatory strategies, but may also expand the molecular genetic arsenal available for research applications in this organism.

\section{MATERIALS AND METHODS}

\subsection{Bacterial Strains and Growth Conditions}

Escherichia coli strains $^{1}$ were grown in Luria-Bertani (LB) broth or agar, supplemented as necessary with kanamycin $(50 \mu \mathrm{g} / \mathrm{ml})$, or oxytetracycline $(10 \mu \mathrm{g} / \mathrm{ml})$. Caulobacter crescentus strains were routinely grown in PYE broth or agar [15], supplemented as necessary with kanamycin $(20 \mu \mathrm{g} / \mathrm{ml}$ in agar, $5 \mu \mathrm{g} / \mathrm{ml}$ in broth), oxytetracycline (2 $\mu \mathrm{g} / \mathrm{ml}$ in agar, $1 \mu \mathrm{g} / \mathrm{ml}$ in broth), and/or nalidixic acid (20 $\mu \mathrm{g} / \mathrm{ml}$ in agar). When indicated, cultures were grown on M2 minimal salts medium [15] containing the carbon/ energy source at a final concentration of $10 \mathrm{mM}$. The Disomer was used for all monosaccharides (galacturonate, glucuronate, glucose, galactose, and xylose). Two sources of pectin were tested: pectin derived from citrus fruits (P9135, Sigma-Aldrich Chemical Co.), and pectin derived from apple (P8471, Sigma-Aldrich Chemical Co.). A final concentration of $1.94 \mathrm{~g} / \mathrm{l}$ pectin was used in media. Growth curves were conducted in $125 \mathrm{ml}$ baffled flasks containing $50 \mathrm{ml}$ PYE or M2 media. Cultures were incubated at $30^{\circ} \mathrm{C}$ in a bench top shaker at $250-300 \mathrm{rpm}$, and samples were taken periodically to monitor turbidity. Phenotype Microarrays (Biolog, Hayward, CA) [16] were also used to examine substrate utilization, as described previously [12]. The PM1 and PM2 plates contain 190 distinct carbon sources, and increases in culture turbidity were used to monitor productive substrate utilization.

${ }^{1}$ Detailed descriptions of bacterial strains, plasmid constructs, and primers are available from the authors upon request.

\subsection{Construction of Mutations}

Knockouts were made by either integrative disruption or non-polar in-frame deletion [17]. Polymerase chain reaction (PCR) primers were synthesized by Integrated DNA Technologies (IDT; San Diego, CA). PCR was done using GoTaq thermostable polymerase (Promega Corp., Madison, WI). Chromosomal DNA was prepared using the DNEasy kit (Qiagen), according to the manufacturer's instructions. Integrative disruptions were constructed as described previously [17], resulting in insertion of the kanamycin-resistant pBGS18T plasmid into the target gene. Construction of non-polar in-frame deletions was done by a PCR-mediated process [17], which results in a deletion of coding sequence and no insertion. For integrative disruptions, the presence of integrated plasmid DNA was verified by PCR. Stability of the integrated plasmids was verified by growth under non-selective conditions (PYE), followed by plating in the absence or presence of kanamycin. After growth under non-selective conditions (PYE) for at least 10 generations, less than 1\% of the population had lost the integrated plasmid-encoded kanamycin resistance. Deletion constructs were also verified by PCR.

\subsection{Electrophoretic Mobility Shift Assays}

C. crescentus strains were grown in $500 \mathrm{ml}$ PYE cultures at $30^{\circ} \mathrm{C}$ to mid-log phase $\left(\mathrm{OD}_{600} \sim 0.4\right)$, and cell lysates were prepared as described previously [13]. Target DNA for binding assays was designed based on the $14 \mathrm{bp}$ HumR operator sequence in the $u x a C$ promoter region. The sequence was slightly modified outside of the putative operator motif to reduce predicted secondary structure, based on mFold [18]. Two 34-base oligonucleotides were synthesized: 5'-CTACGCGCGATGACACCGGTTACCAGCAGACACG and 5'-CGTGTCTGCTGGTAACCGGTGTCATCGCGCGTAG (putative HumR operator underlined). The 5' end of each was labeled with Cy5 (IDT). Oligonucleotides were annealed in equimolar amounts by first denaturating at $95^{\circ} \mathrm{C}$ for $5 \mathrm{~min}$, then cooling slowly for 60 minutes to room temperature. Binding reactions consisted of $2.5 \mathrm{ng}$ annealed duplex target DNA, binding buffer $(150 \mathrm{mM} \mathrm{KCl}, 0.1 \mathrm{mM}$ dithiothreitol, $0.1 \mathrm{mM}$ EDTA and $10 \mathrm{mM}$ Tris (pH 7.4)), $1 \mu \mathrm{g}$ poly$\mathrm{dI}: \mathrm{dC}$, and $22.5 \mu \mathrm{g}$ protein in a total reaction volume of $15 \mu \mathrm{l}$. For binding inhibition assays, galacturonate, glucuronate, glucose, xylose, or galactose were added to 10 $\mathrm{mM}$ final concentration as appropriate. Reactions were incubated at room temperature for 10 minutes, and then resolved on a $6 \%$ non-denaturing polyacrylamide gel made and run with $1 \times$ Tris-borate-EDTA buffer. Gels were run at $100 \mathrm{~V}$ for $50 \mathrm{~min}$, visualized on a Typhoon imaging system (Molecular Dynamics) and quantified using Image Quant software. Background counts were deter- 
mined in lanes where no protein was added, and subtracted from the counts for lanes that contained cell extracts.

\subsection{Expression Assays and Operator Mutants}

To examine regulation of gene expression in response to galacturonate, the regions upstream of the CC_1488 (uxaA) and CC_1490 (uxaC) genes, respectively, were amplified by PCR. For wild-type promoters, PCR primers were designed to amplify up to $250 \mathrm{bp}$ upstream of the start codons of these genes, along with a few hundred base pairs within the coding region. Synthetic EcoRI or HindIII sites were added to the 5' end of the primers to allow for directional cloning. PCR products were initially cloned into plasmid PCR2.1 using the TOPO Cloning System (Life Technologies), then subcloned into pRKlac290, a low copy-number, broad host range plasmid to generate transcriptional fusions to the lac $Z$ gene [19]. pRKlac290 constructs were transferred from E. coli S17.1 by conjugation into $C$. crescentus strains, with selection on PYE supplemented with nalidixic acid and oxytetracycline. Construction of targeted mutations in the putative operator for the $u x a C$ promoter was done with a two-step PCR-based method [17]. Promoter constructs containing the mutant operators were cloned as described above, and $\beta$-galactosidase activity was assayed as described previously $[13,19]$.

\section{5. qRT-PCR Analysis of Gene Expression}

Quantitative real-time PCR (qRT-PCR) was used to examine gene expression, starting with RNA isolated from C. crescentus strains grown in PYE medium, with or without galacturonate. RNA was extracted as described previously [11]. Isolated RNA was subjected to two consecutive genomic DNA removal treatments by digesting with $2 \mathrm{U}$ DNaseI (Ambion) per $10 \mu \mathrm{g}$ RNA, incubated at $37^{\circ} \mathrm{C}$ for $60 \mathrm{~min}$. DNaseI was inactivated by addition of phenol:chloroform (5:1) and RNA was precipitated with $3 \mathrm{M}$ sodium acetate $(\mathrm{pH} 5.2)$ and $100 \%$ ethanol at $-20^{\circ} \mathrm{C}$ overnight. Pellets were obtained by centrifugation at $16,000 \times \mathrm{g}$ for $15 \mathrm{~min}$, washed with $70 \%$ ethanol and resuspended in RNase free water. RNA purity was determined spectrophotometrically and agarose gels were run to verify RNA integrity.

Complementary DNA (cDNA) was synthesized with $2.0-2.5 \mu \mathrm{g}$ of RNA and SuperScriptII Reverse Transcriptase (200 units, Life Technologies) with random primers (100 ng per $5 \mu \mathrm{g}$ total RNA, Life Technologies) following the manufacturer's suggested protocol, including the optional RNaseH digestion (New England Biolabs). Primer and probe sequences for qRT-PCR were designed using the PrimerExpress v3.0 software (Life Technologies) and synthesized by IDT. Because primers did not include the minor grove binder, the probe $\mathrm{T}_{\mathrm{m}}$ parameter was adjusted to be $10^{\circ} \mathrm{C}$ higher than the primer $\mathrm{T}_{\mathrm{m}}$ values. Analyses for heterodimer and homodimer formation and secondary structure were done using IDT's OligoAnalyzer 3.1, and BLAST was used to examine the potential for non-target site primer/probe annealing. Specificity of primers and probes was subsequently verified by analyzing qRT-PCR products on agarose gels. Probes were labeled with the 5' reporter dye 6-FAM and the 3' quencher Iowa Black FQ. qRT-PCR was performed on a StepOne Real Time PCR System (Life Technologies) in $20 \mu \mathrm{l}$ reaction volumes consisting of $1 \times$ TaqMan Gene Expression Master Mix, $900 \mathrm{nM}$ forward and reverse primers, $250 \mathrm{nM}$ probe, and $500 \mathrm{ng}$ of cDNA. Thermocycling conditions were: $95^{\circ} \mathrm{C}$ for $10 \mathrm{~min}$, followed by 40 cycles of $95^{\circ} \mathrm{C}$ for $15 \mathrm{sec}$ and $60^{\circ} \mathrm{C}$ for $60 \mathrm{sec}$. Amplification efficiency of each primer and probe set was determined using a standard curve. The five dilutions of the standard curve were tested in triplicate and were serially diluted five-fold from a starting quantity of $125 \mathrm{ng} / \mu \mathrm{l}$. In each experiment, samples were run in duplicate and two internal controls were used to normalize results [20]. The genes used for normalization were rpoA (CC_1272), which encodes the RNA polymerase alpha subunit, and secA (CC_3068), which encodes the pre-protein translocase subunit SecA. These controls were chosen based on expected constitutive expression under all conditions used, and because their functions are unrelated to the genes under investigation. In addition, raw RNA extract and CB15 genomic DNA were included to detect the presence of contaminating genomic DNA.

Threshold levels for each gene were adjusted to control for inter-assay variation and the relative expression of each gene was determined with the comparative $\Delta \Delta C_{T}$ method [21]. The $\Delta \mathrm{C}_{\mathrm{T}} \mathrm{s}$ for each sample and target were first obtained by normalizing the threshold cycle number $\left[\mathrm{C}_{\mathrm{T}}\right]$ to the mean $\mathrm{C}_{\mathrm{T}}$ of constitutively expressed rpo $A$ and $\sec A$ genes. Relative quantification (RQ) values were calculated using the equation $\mathrm{RQ}=(1+\mathrm{E})^{-\Delta \Delta \mathrm{CT}}$, where $\mathrm{E}$ is the amplification efficiency of each target determined by a standard curve and $\Delta \Delta \mathrm{C}_{\mathrm{T}}=\Delta \mathrm{C}_{\mathrm{T} \text { reference }}-\Delta \mathrm{C}_{\mathrm{T} \text { sample }}[22]$.

\subsection{RT-PCR of Hexuronate Metabolism Genes}

To determine whether closely spaced genes involved in galacturonate metabolism are encoded on the same transcript, reverse transcriptase-mediated PCR was employed for the following gene pairs: CC_1487-1488 (uxaB and uxaA), CC 1490-1491 (uxa $\bar{C}$ and $k d u I$ ), and CC_1495-1496 $(\overline{k d g} A$ and $k d g K)$. Primers were designed with the forward primer annealing $150-300 \mathrm{bp}$ upstream of the 3 ' end of the first gene, and the reverse primer annealing in the coding region of the putative linked downstream gene. As a control, primers were designed for the 
CC_3040-3041 gene pair (fliI and fliJ), which are known to be co-transcribed in a polycistronic mRNA. RNA samples were prepared as described above and used as a template for cDNA synthesis. CB15 genomic DNA was included as a positive control. Templates were diluted to $100 \mathrm{ng} / \mu \mathrm{l}$ and PCR was performed in $25 \mu \mathrm{l}$ reaction volumes containing 1× Green GoTaq Buffer (Promega), $0.25 \mathrm{mM}$ dNTPs, $1 \mu \mathrm{m}$ each forward and reverse primer, 0.75 U GoTaq DNA Polymerase (Promega) and $1 \mu \mathrm{ltem-}$ plate. Thermocycling conditions were: $95^{\circ} \mathrm{C}$ for $2 \mathrm{~min}$, followed by 30 cycles of $95^{\circ} \mathrm{C}$ for $15 \mathrm{sec}, 60^{\circ} \mathrm{C}$ for $25 \mathrm{sec}$, and $72^{\circ} \mathrm{C}$ for $50 \mathrm{sec}$, with a final $5 \mathrm{~min}$ extension at $72^{\circ} \mathrm{C}$. Products were visualized by agarose gel electrophoresis using $0.9 \%$ agarose, and the gels were subsequently stained with ethidium bromide.

\section{RESULTS}

\subsection{Growth of $C$. crescentus on Hexuronates}

Analysis of the C. crescentus CB15 genome sequence suggested that it encodes enzymes for the degradation of several major plant polysaccharides, including cellulose, xylan, lignin, glucan, and pectin [8]. To investigate pectin and hexuronate utilization, growth of $C$. crescentus was examined using pectin, galacturonate, and glucuronate as sole carbon sources. (The D-isomer of all monosaccharides was used in this work.) Pectin produced mixed results. Under conditions in which glucose produced robust colonies in 3 days, pectin derived from apple peel yielded small colonies in 6 days, and pectin derived from citrus fruit did not support visible growth. Pectin from different plant sources can differ significantly in structure and digestibility [1], but the results with apple peel pectin show that $C$. crescentus is capable of consuming at least some forms of this polymer. The core pectin chain is comprised of alpha-1,4-linked galacturonate. Pure galacturonate $(10 \mathrm{mM})$ supported growth of $C$. crescentus on plates, with small colonies visible after 4 - 5 days. In liquid M2 medium, the log phase generation time on galacturonate was approximately 4.3 hours, roughly twice as long as doubling times on glucose $(2.0 \mathrm{~h})$ or xylose $(2.3 \mathrm{~h})$. Adaptation to growth on galacturonate was slow; wild-type cells transitioning from glucose to galacturonate in M2 medium experienced a prolonged lag phase of $24 \mathrm{~h}$ or more before initiating exponential growth, roughly three-fold longer than the lag phase in transitioning from glucose to xylose. Growth on glucuronate was comparable to growth on apple pectin, suggesting that $C$. crescentus "prefers" galacturonate over glucuronate.

\subsection{Genetic Characterization of Galacturonate Utilization}

In bacteria, pectate (demethylated pectin) can be cleaved by pectatelyase and/or exo-poly-galacturonidase to gen- erate oligo-galacturonides and galacturonate for import to the cytoplasm [2]. Two bacterial pathways have been identified for galacturonate metabolism [3]: the hexuronate isomerase pathway studied extensively in E. coli and E. chrysanthemi, and the oxidative pathway active in $A g$ robacterium tumifaciens and certain Pseudomonas species. Although $C$. crescentus is much more closely related to A. tumifaciens (an alpha-Proteobacterium) than to $E$. coli or E. chrysanthemi (gamma-Proteobacteria) [23], the $C$. crescentus genome contains a set of genes encoding the gamma-Proteobacterial hexuronatei somerase pathway, and lacks a close relative of the galacturonate dehydrogenase enzyme that is key to the A. tumifaciens pathway [24]. The genomic region containing the genes of the putative $C$. crescentus hexuronate metabolism pathway is shown in Figure 1. This gene cluster is likely organized as several operons, based on orientation and gene spacing (Figure 1(A)). Reverse transcriptase-mediated PCR confirmed that CC_1488 and CC_1487 are transcribed on the same mRNA, as are CC $-1490-1491-1492$, and CC_1495-1496 (data not shown). Figure 1(B) shows the hexuronate isomerase pathway enabled by the products of this gene cluster $[3,5]$. The penultimate step generates 2keto-3-deoxy-6-phospho-D-gluconate, an intermediate shared with the Entner-Doudoroff pathway, the sole route of glucose catabolism in C. crescentus [11,25]. The final step, catalyzed by keto-deoxyphosphogluconate aldolase (KdgA, CC_1495 gene product), yields pyruvate and glyceraldehyde-3-phosphate. We have shown previously that CC_1495 is necessary for glucose metabolism [11].

To determine whether genes in this cluster are necessary for growth on galacturonate, a series of mutant strains were constructed and tested for growth on M2 media using galacturonate as sole carbon and energy source (Table 1). As controls, the mutant strains were also tested for growth on glucose and xylose. Strains containing mutations in the CC_1487 (uxaB), CC_1488 (uxaA), CC_1490 (uxaC), and CC_1495 (kdgA) genes were incapable of growth on galacturonate. The $k d g A$ disruption mutant, like the strains with point mutations in $k d g A$ investigated previously, is also incapable of growth on glucose, as the encoded enzyme catalyzes an essential step in the Entner-Doudoroff pathway. Mutations in genes encoding enzymes catalyzing "upstream" reactions in the Entner-Doudoroff pathway (CC_2054-2057) [11] blocked glucose utilization, but had no effect on galacturonate metabolism. The $u x a B$, uxaA, and $u x a C$ mutants grow normally on glucose and xylose, and the only growth defects relative to wild-type C. crescentus apparent on Biolog Phenotype Microarrays were with galacturonate and glucuronate. (Pectin did not support significant growth of C. crescentus in this assay.) Strains containing mutations in CC_1491 $(k d u I)$ and CC_1492 $(k d u D)$ grew normally on galacturonate, consistent with their inferred role in the 
(a)

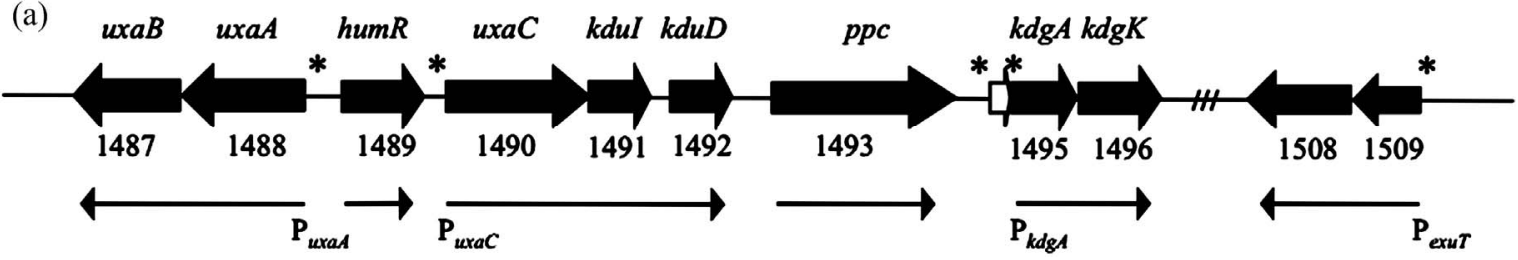

(b)

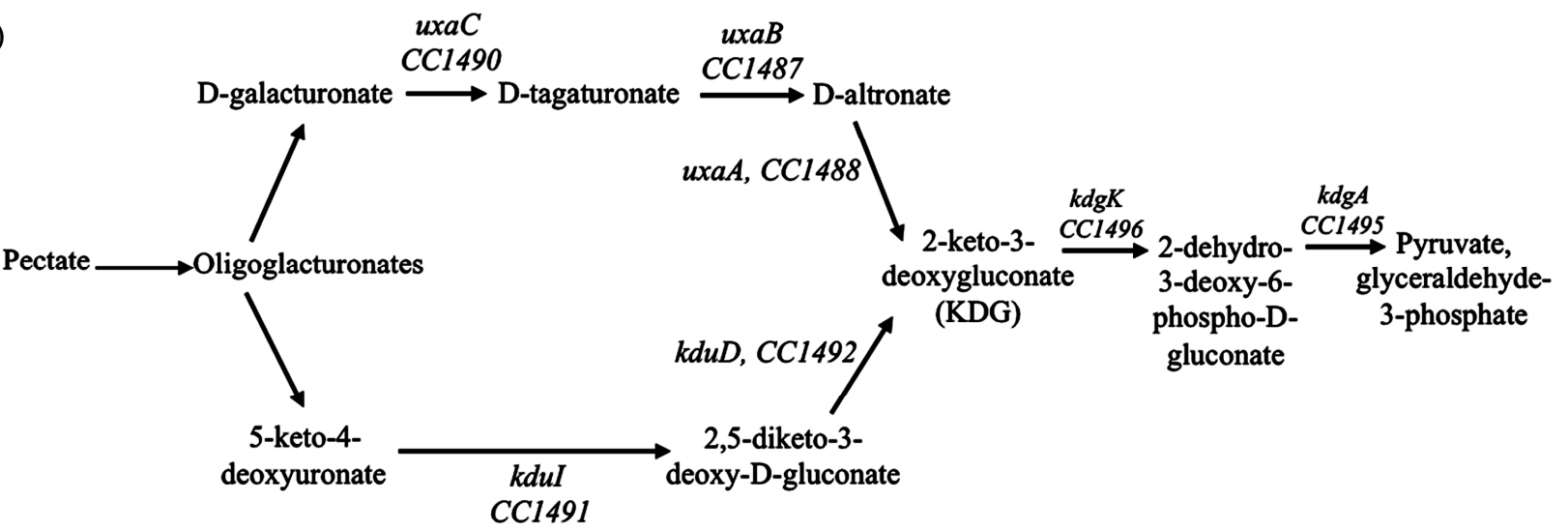

Figure 1. A putative pathway for D-galacturonate degradation in C. crescentus. A)The CC_1487-1509 genome region in C. crescentus $\mathrm{CB} 15$. The CC 1494 gene predicted in the original $C$. crescentus genome annotation is shown in white. The putative CC_1494 ORF (128 codons) has no significant relatives in GenBank. The CC_1497 to CC_1507 region is not shown [hash marks] as their predicted functions do not appear to be connected to hexuronate utilization; B) Proposed C. crescentus pectin/hexuronate degradation pathway.Polygalacturonides derived from pectin are degraded to yield monomeric galacturonate or 5-keto-4-deoxyuronate, which are processed through parallel pathways merging at 2-keto-3-deoxygluconate (KDG). The C. crescentus genes hypothesized to encode the enzyme responsible for each step in the pathway are indicated. Gene names are based on homology to genes of known function in E. coli and E. chrysanthemi.

Table 1. Growth of $C$. crescentus strains containing mutations in genes encoding putative enzymes of the galacturonate metabolic pathway. "-" indicates that no individual colonies formed on M2 minimal salts agar with the indicated sole carbon and energy source within 7 days. " \pm " indicates that colonies less than half the size of the wild-type control after 7 days. "+" indicates colonies that formed at a similar rate and size to wild-type.

\begin{tabular}{|c|c|c|c|c|}
\hline \multirow[b]{2}{*}{ Disrupted gene } & \multirow[b]{2}{*}{ Annotated function } & \multicolumn{3}{|c|}{ Growth with sole carbon/energy source: } \\
\hline & & Galacturonate & Glucose & Xylose \\
\hline CC_1487 uxaB & D-tagaturonate reductase & - & + & + \\
\hline CC_1488uxaA & D-altronate dehydratase & - & + & + \\
\hline CC_1489 humR & Transcriptional regulator, LacI-GalR family & + & + & + \\
\hline CC_1490 uxaC & Glucuronate isomerase & - & + & + \\
\hline CC_1491 kduI & 5-keto-4-deoxyuronate isomerase & + & + & + \\
\hline CC_1492 kduD & 2-deoxy-D-gluconate 3-dehydrogenase & + & + & + \\
\hline CC_1493 ppc & Phosphoenolpyruvate carboxylase & - & - & + \\
\hline CC_1495 kdgA & 2-keto-3-deoxyphosphogluconate aldolase & - & - & + \\
\hline CC_1496 kdgK & 2-dehydro-3-deoxyglucokinase & \pm & \pm & + \\
\hline
\end{tabular}

parallel branch that processes 5-keto-4-deoxyuronate, a product of pectate lyase.

The CC_1496 mutant strain grew slower on galacturonate relative to wild-type, but still formed detectable colonies, indicating that this gene product is important but not essential for galacturonate metabolism. We des- ignated CC_1496 as $k d g K$, based on its genome location and weak sequence similarity to KdgK (2-keto-3-deoxygluconate kinase) gene products, but the knockout results suggest that it may not be the only gene product able to carry out this phosphorylation reaction.

The $p p c$ gene (CC_1493) is necessary for growth of $C$. 
crescentus on glucose and galactose [11], and was found here to be essential for growth on galacturonate. $\mathrm{CC}_{-}$ 1493 encodes phosphoenolpyruvate (PEP) carboxylase, which catalyzes the addition of $\mathrm{CO}_{2}$ to PEP to generate oxaloacetate. During growth on minimal media with glucose or galacturonate as the sole carbon input, PEP carboxylase may serve an anaplerotic function, replenishing oxaloacetate levels to allow the TCA cycle to continue as intermediates are withdrawn for use in other biosynthetic pathways.

\subsection{Regulation of Galacturonate Metabolism}

To determine whether expression of genes implicated in galacturonate metabolism is induced in response to galacturonate in the culture medium, the regions upstream of uxaA (CC_1488) and uxaC (CC_1490) were inserted next to a promoterless $l a c Z$ reporter on a low copy-number plasmid vector. $\beta$-galactosidase activity was assayed in wild-type $C$. crescentus grown in PYE medium [15] before and after exposure to $10 \mathrm{mM}$ galacturonate, glucuronate, or other sugars. In plain PYE, promoter activity was very low in both constructs $\left(\mathrm{P}_{u x a A}=14 \pm 24\right.$ Miller units, $\mathrm{P}_{u x a C}=54 \pm 29$ ). In the presence of galacturonate, promoter activity increased dramatically $\left(\mathrm{P}_{\text {uxaA }}=239 \pm\right.$ 139 , 17-fold increase; $\mathrm{P}_{u x a C}=1150 \pm 214,21$-fold increase). Subsequent analysis by quantitative real-time PCR (qRT-PCR) showed that both promoters were fully induced within 5 minutes of exposure to galacturonate. $\mathrm{P}_{\text {uxaA }}$ was relatively weak compared to $\mathrm{P}_{\text {uxaC }}$, but the behavior of the two promoters was qualitatively similar. Glucuronate also induced expression of both promoters, though somewhat less effectively than galacturonate $\left(\mathrm{P}_{u x a A}=15\right.$-fold increase; $\mathrm{P}_{u x a C}=5$-fold increase $)$. Neither glucose, galactose, or xylose induced either promoter, nor did they prevent induction of these promoters by galacturonate. Expression of these promoters was also examined in cultures grown in M2 medium, with similar results (data not shown).
The CC_1489 gene, located in the midst of the hexuronate metabolism gene cluster, encodes a member of the LacI repressor family. The polypeptide sequence encoded by CC_1489 is similar to C. crescentus XylR (CC 3065; 38\% identity over 438 amino acids with CC_ 1489 ), which controls a gene set for xylose metabolism $[11,13]$. To determine whether CC_1489 controls genes involved in galacturonate metabolism, an integrative disruption was constructed. The disruption mutant exhibited a log phase growth rate virtually identical to wild-type on M2 minimal medium supplemented with 10 $\mathrm{mM}$ glucose, xylose, galacturonate, or glucuronate, but transitioned more quickly to logarithmic growth when transferred from glucose to galacturonate or glucuronate. When expression of the $u x a A$ and $u x a C$ promoters $\left(\mathrm{P}_{u x a A}\right.$ and $\mathrm{P}_{u x a C}$, respectively) was examined in the $\mathrm{CC}_{-} 1489$ disruption mutant, both became highly active under noninducing conditions, exceeding the activity of the promoters in wild-type CB15 in the presence of galacturonate (Table 2 line 1; $\mathrm{P}_{\text {uxaA }}$ results not shown). In the CC 1489 mutant background, addition of galacturonate did little to increase promoter activity beyond that in plain PYE. These observations are consistent with the CC_1489 product acting as a repressor of $\mathrm{P}_{u x a A}$ and $\mathrm{P}_{u x a C}$ in the absence of galacturonate. We therefore have designated the CC_1489 gene product HumR, for hexwronate metabolism regulator.

MEME [26] was used to search for conserved sequences in the intergenic regions of the CC_1487-1496 cluster, to identify candidate binding sites for potential regulatory factors. The annotated CC_1494 coding region was included in the search, since this small ORF is not conserved at the protein level in any other species and we suspect that it may not actually encode a polypeptide. A conserved $14 \mathrm{bp}$ sequence (TGACACC) GGTTACC) was identified upstream of the $u x a A, u x a C$, and $k d g A$ genes in the $C$. crescentus genome, and in similar locations upstream of these genes in C. henricii K31,

Table 2. Regulation of $\mathrm{P}_{u x a c}$-lacZ expression by HumR and galacturonate. Promoter constructs with identical 5' and 3' ends and sequences, save for the mutations shown, were inserted upstream of a promoterless lac $Z$ gene in the low copy number pRKlac290 reporter vector. The resulting $\beta$-galactosidase activities (Miller units) are shown in the table.

\begin{tabular}{|c|c|c|c|c|c|c|c|}
\hline \multirow[b]{2}{*}{ Promoter } & \multirow[b]{2}{*}{ Operator } & \multicolumn{3}{|c|}{ CB15 (wild-type) } & \multicolumn{3}{|c|}{ humR disruption } \\
\hline & & PYE & PYE + gal & Ratio (+/- gal) & PYE & PYE + gal & Ratio (+/- gal) \\
\hline $\mathrm{P}_{u x a C}$ & TGACACCGGTTACC & $52 \pm 28$ & $1090 \pm 165$ & 21 & $3200 \pm 54$ & $4900 \pm 498$ & 1.5 \\
\hline Mut A & TGACA $\underline{G A G G T T A C C}$ & $3310 \pm 208$ & $3540 \pm 374$ & 1.1 & $3610 \pm 132$ & $3160 \pm 177$ & 0.9 \\
\hline Mut B & TGA $\underline{\text { GGCCGGTTACC }}$ & $4810 \pm 891$ & $4520 \pm 578$ & 0.9 & $4970 \pm 191$ & $4410 \pm 112$ & 0.9 \\
\hline Mut C & TGACACC $\underline{\text { TAGTACC }}$ & $2810 \pm 692$ & $2580 \pm 287$ & 0.9 & $2500 \pm 280$ & $2250 \pm 289$ & 0.9 \\
\hline Mut D & 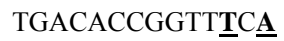 & $43+15$ & $85 \pm 4$ & 2.0 & $-{ }^{1}$ & $-{ }^{1}$ & $-{ }^{1}$ \\
\hline
\end{tabular}

${ }^{1}$ Promoter activity was not detectable, as measured $\beta$-galactosidase activity was at or below background levels for the parental strain. Because of this, some activity ratios could not be calculated for this construct. 
Caulobacter segnis, P. zucineum, and A. excentricus. To determine whether this motif is relevant to regulation, four distinct multi-base mutations were generated in the motif upstream of $C$. crescentus uxaC to examine their effects on gene expression and regulation (Table 2). With three of the mutations (Tables 2(A)-(C)), $\mathrm{P}_{\text {uxaC }}$ was expressed at high levels in the absence of galacturonate, and was not further activated by genetic elimination of HumR. These results are consistent with an operator site responsible for interaction with a repressor. By contrast, "MutD", a 2 bp change in the far right of the putative operator sequence, virtually eliminated $\mathrm{P}_{u x a C}$ expression under inducing conditions. A possible explanation for this is presented in the "Discussion" section.

To determine whether HumR binds to the conserved operator, an electrophoretic mobility shift assay was carried out using a fluorescently-labeled synthetic uxaC operator. Crude extracts from wild-type $C$. crescentus formed a distinct shifted complex with the synthetic operator (Figure 2, lane 2) that was virtually eliminated when extracts from the humR mutant were used (Figure 2 , lane 3). In the presence of $10 \mathrm{mM}$ galacturonate or glucuronate, the intensity of the wild-type complex was greatly reduced (Figure 2, lanes 4 and 5). Glucose and galactose had no effect on the complex, demonstrating specificity of HumR for hexuronates. These observations are consistent with a LacI-type model in which HumR loses affinity for the operator when inducer (galacturonate or glucuronate) is present, allowing transcription to proceed. A faint lower-molecular weight complex was also seen that was present for all strains tested, and with all sugars. This band may reflect another protein able to

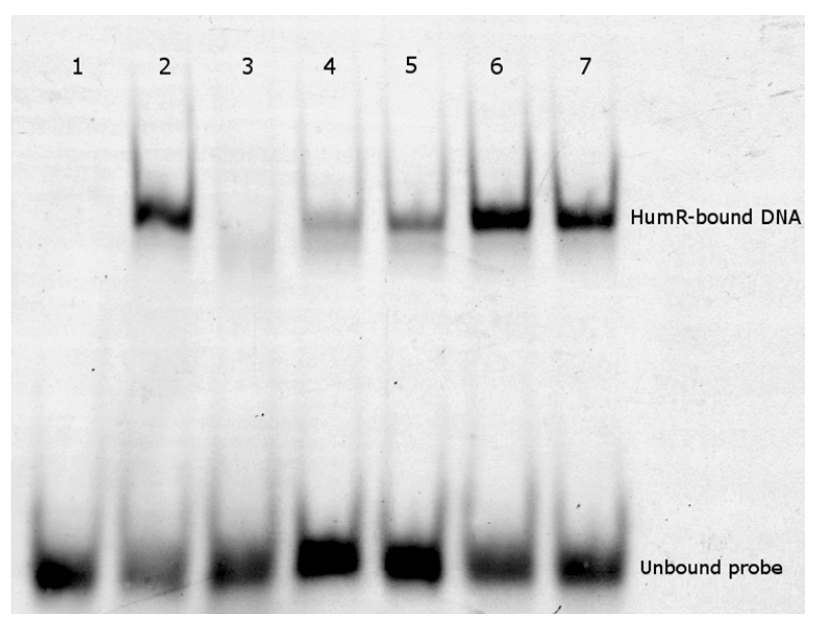

Figure 2. Gel mobility shift assay demonstrating HumR binding to a synthetic $\mathrm{P}_{u x a C}$ operator. Lane 1 , no cell extract added; lane 2, CB15 cell extract; lane 3, humR mutant cell extract; lane 4, CB15 cell extract with $10 \mathrm{mM}$ galacturonate; lane 5, CB15 cell extract with $10 \mathrm{mM}$ glucuronate; lane $6, \mathrm{CB} 15$ cell extract with $10 \mathrm{mM}$ galactose; lane $7, \mathrm{CB} 15$ cell extract with $10 \mathrm{mM}$ glucose. interact weakly with the synthetic probe.

\subsection{The HumR Regulon}

To identify other genes potentially regulated by HumR, the MEME-identified conserved motif was used to search the C. crescentus genome [8]. After filtering the results to focus only on sequences in intergenic regions, several potential operators were identified (Table 3). Within the CC_1487-1495 cluster, a second potential operator sequence upstream of CC_1494-1495 shared 11 out of 14 bp. Other potential operators included a 14/14 bp match located $116 \mathrm{bp}$ upstream of the start codon for CC_1446, the first gene in a three gene unit (CC_1446-1448) annotated as encoding the three key components of an inner membrane TRAP family transport system that could potentially serve as an ATP-independent transporter for hexuronates [27]; a 13/14 bp match located 79 bp upstream of CC_1509, the first gene in a likely operon that includes a homolog of the exuT hexuronate transporter (CC_1508) [28]; an 11/14 bp match located 99 bp upstream of the start codon for CC_3152, which is annotated as encoding a pectatelyase; and an 11/14 bp match located upstream of CC_0442, a putative TonB-dependent outer membrane receptor.

To determine whether other potential HumR operators can convey HumR- and galacturonate-dependent regulation of transcription, expression of CC_1446, CC_1509, and CC_3152 was assayed by qRT-PCR. Because the gene expression analyses presented earlier were based on lac $Z$ fusions, we also examined $u x a A$ and $u x a C$ expression by qRT-PCR. RNA was obtained from both wild type and humR mutant strains grown in PYE medium in the presence or absence of galacturonate. The cultures all had very similar growth rates and were harvested at similar densities. All of the genes with potential HumR operators showed induction by galacturonate, and were activated in the humR mutant strain in the absence of galacturonate (Table 4). It is not clear why the apparent magnitude of regulation for $\mathrm{P}_{u x a A}$ and $\mathrm{P}_{u x a C}$ varies substantially when assayed by different techniques. Effective qRT-PCR primer and probe sets could not be designed for CC0442 and CC1494, but microarray data (unpublished) has shown that expression of these mRNA's is strongly regulated by HumR and galacturonate, placing them in the HumRregulon as well.

\section{DISCUSSION}

\subsection{Utilization of Pectin and Galacturonate}

Caulobacter species are not known to act as plant pathogens, but likely scavenge decaying plant biomass in aquatic environments. The data presented here show that C. crescentus is capable of consuming at least some 
Table 3. Possible HumR operator sequences upstream of genes potentially involved in galacturonate acquisition, transport, and degradation. The sequences shown are all in intergenic regions. See "Results" section for more on predicted functions of each gene.

\begin{tabular}{cccc}
\hline Adjacent gene & Putative operator sequence & Orientation of operator relative to promoter & Distance from start of operator to translational start [bp] \\
\hline CC_1488 & TGA CAC CGG TTA CC & + & 69 \\
CC_1490 & TGA CAC CGG TTA CC & + & 75 \\
CC_1495 & TGA CAC CGG TTA CC & - & 385 \\
CC_1495 & TGA CAC CGG TGG CA & + & $435^{1}$ \\
CC_0442 & TGA CAC CGG TGG CA & + & 129 \\
CC_1446 & TGA CAC CGG TTA CC & + & 115 \\
CC_1509 & TGA CAC CGG TTT CC & + & 78 \\
CC_3152 & TGA CAC CGG TGGAC & + & 96 \\
\hline
\end{tabular}

${ }^{1}$ Distance to the annotated CC_1494 translational start is $44 \mathrm{bp}$. As described in the legend to Figure 1, the legitimacy of CC-1494 as a functional coding region is uncertain.

Table 4. qRT-PCR analysis of gene expression and regulation by HumR and galacturonate. Strains used were wild-type CB15, and a humR disruption mutant in the CB15 background. Cultures were grown in PYE medium, and RNA was isolated from cultures at $\mathrm{OD}_{600 \mathrm{~nm}} \sim 0.3$. "Induced" cultures had galacturonate added to $10 \mathrm{mM}$ final concentration 1 hour before the culture reached the target density. See "Results" section for more on predicted functions of each gene.

\begin{tabular}{ccc}
\hline Gene & humR $/$ WT ratio & Induced/uninduced ratio \\
\hline CC_1488 & $53.2 \pm 19.8$ & $7.00 \pm 2.85$ \\
CC_1490 & $50.0 \pm 25.9$ & $6.51 \pm 2.79$ \\
CC_1446 & $2.48 \pm 0.51$ & $2.01 \pm 0.41$ \\
CC_1509 & $111 \pm 30$ & $73.8 \pm 20.5$ \\
CC_3152 & $14.1 \pm 2.2$ & $9.14 \pm 1.46$ \\
\hline
\end{tabular}

forms of pectin, and grows well on galacturonate, pectin's core monosaccharide. Genetic evidence indicates that C. crescentus metabolizes galacturonate via a pathway starting with hexuronateisomerase, while a parallel pathway processes 5-keto-4-deoxyuronate. The two branches start with distinct pectin breakdown products, probably to accommodate the use of both pectate lyase and polygalacturonidase. Analysis of the $C$. crescentus genome shows two putative pectate lyases (CC_3152 and CC_2035) and a putative exo-poly-alpha-galacturonosidase ("exoPG”, CC_0572), each predicted by SignalP to contain an $\mathrm{N}$-terminal signal peptide whose presence is consistent with secretion. In E. chrysanthemi, exo-PG and pectatelyase are coordinately regulated, and are both needed for maximally efficient breakdown of pectin [29]. Whether this is true in $C$. crescentus requires further investigation.

The optimization of $C$. crescentus for utilization of galacturonate, rather than glucuronate, would be sensible if galacturonate, as a component of pectin, is more abundant than glucuronate in niches this freshwater microbe is likely to inhabit. The underlying physiological explanation for this adaptation would require comprehensive analysis of transport, regulation, and enzymatic activities. Since glucuronate is capable of inducing the HumR regulon, it presumably is imported into the cell, suggesting that the reason for inefficient growth is due to differential metabolism in the cytoplasm.

\subsection{Regulation by HumR}

Expression of several genes implicated in galacturonate utilization in $C$. crescentus is controlled by HumR, a member of the LacI family of bacterial transcription factors [30]. There are 11 LacI family members encoded in the $C$. crescentus genome. Other are known to control uptake and/or metabolism of xylose (CC_3065), glucose (CC_2053), and maltose (CC_2284) [11,13,21]. Data presented here indicates that HumR binds to cognate operators in the absence of galacturonate, and interferes with effective RNA polymerase interaction with the respective promoter. The data are consistent with galacturonate binding to HumR, stabilizing a conformation with reduced affinity for operator DNA and releasing it from the promoter region to allow gene expression. Glucuronate also appears to productively interact with HumR as well, both in vitro and in vivo.

HumR functions similarly to the $C$. crescentus xylose repressor, XylR [13]. The XylR regulon includes a xylose transporter and an operon encoding enzymes for xylose metabolism, as well as putative secretive xylanases and arabinosidases [11]. The HumR operator (TGACACC| GGTTACC) and XylR operator (TGTTAGC|GCTACCA) sequences are the same length (14 bp), and identical at 7 out of 14 positions [13]. Given that LacI-type repressors bind as dimers, dyad symmetry is expected in the operator. All of the identified HumR operators are identical in 
one half-site (TGACACC), which we suspect may be the optimal binding sequence for a HumR subunit. We have been unable to experimentally determine the 5 ' end of a HumR-regulated transcript, but alignment of $\mathrm{P}_{u x a C}$ sequences from the Caulobacter group (Figure 3(a)) reveals additional conserved sequences flanking the operator that match the -10 and -35 elements of $\mathrm{P}_{x y l}$ (Figure 3(b)), for which the 5' end is known. In $\mathrm{P}_{x y l}$, the XylR operator is positioned between the -35 and -10 regions, overlapping the predicted downstream contact site for the $\sigma$ submit of RNA polymerase. If the aligned sequences represent the same functional promoter elements in $\mathrm{P}_{x y l}$ and $\mathrm{P}_{u x a C}$, then bound HumR would likewise overlap the -10 region, allowing it to block critical contacts with the $\sigma$ subunit. If that is true, the sequence of the right half-site of the HumR operator in $\mathrm{P}_{\text {uxaC }}$ is probably under dual selection for binding HumR and $\sigma$. This is consistent with the effect of OpMutD, a 2 bp change on the far right side of the operator (Table 2) that actually makes the sequence of the HumR operator half site more similar to the left side consensus, but changes the putative -10 region away from that shared with $\mathrm{P}_{x y l}$. Table 2 shows that promoter activity was dramatically reduced by this mutation, even under conditions where HumR binding should be eliminated, supporting the hypothesis that the operator overlaps with a critical promoter element.

As with the XylR regulon, some HumR-regulated promoters show mild reductions in inducibility when glucose is present, but glucose does not preclude induction of XylR or HumR-regulated promoters. There are no significantly conserved sequence elements in these promoters other than the identified operators, so direct involvement of additional glucose-responsive transcription factors such as CAP (E. coli) or CcpA (Bacillus subtilis kduID operon [31]) seems unlikely. As an oligotroph adapted to low-nutrient habitats, there may be minimal reward for $C$. crescentus to prioritize glucose utilization at the expense of other available sugars.

The HumR regulon in $C$. crescentus includes at least five inferred operons (CC_1488-1487, CC_1490-14911492, CC_1495-1496, CC_1509-1508 and CC_14461448 ) and two singleton genes (CC 3152 and CC 0442 ). Based on annotated functions, the components of the HumR/galacturonate regulon fit the pattern previously established for the XylR/xylose regulon [11,13], and for glucose-dependent gene expression [11], in that they all include extracellular polysaccharide-active enzymes, TonBdependent outer membrane receptors (TBDR's), inner membrane transport systems, and a cytoplasmic catabolic pathway. Thus, each repressor likely controls a complete system for extracellular polysaccharide degradation, uptake of breakdown products, and intracellular catabolism of monosaccharides to yield energy and central metabolites. The use of TBDR's for scavenging polysaccharide degradation products is shared by phytopathogenic Xanthomonas species, and Blanvillain et al. [32] recognized that TBDR's tend to be genetically clustered with "carbohydrate active enzymes" and inner membrane transport systems to create "CUT" (carbohydrate-utilization) loci. Caulobacter crescentus apparently did not maintain tight genetic linkage of CUT systems over evolutionary time, but still coordinates expression of components through repressors that recognize specific monosaccharides as induction signals.

In E. coli and Erwinia chrysanthemi, pectin degradation and hexuronate utilization are primarily controlled by the ExuR, UxuR, and KdgR transcription factors spe-

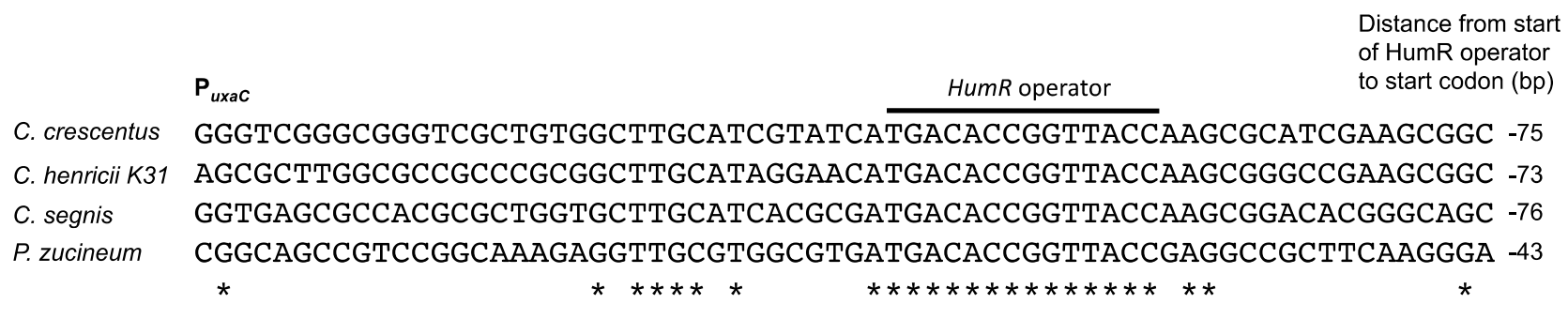

(a)

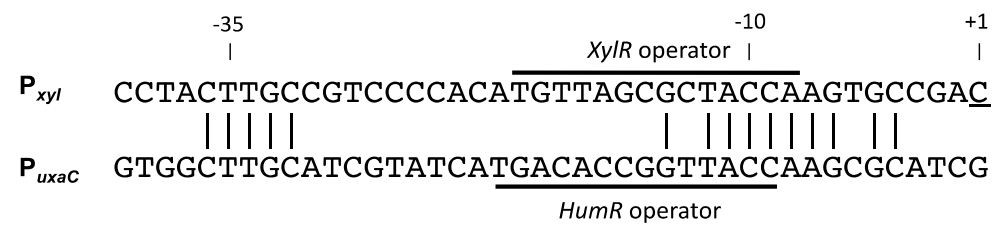

(b)

Figure 3. The $u x a C$ promoter and HumR operator. a) Alignment of $u x a C$ promoter region in C. crescentus and related species $(C$. henricii K31, C. segnis and P. zucineum). Distance is shown to the start codon, as transcription start sites could not be determined; b) Alignment of C. crescentus $\mathrm{P}_{x y l}$ [14] and $\mathrm{P}_{u x a C}$. Numbering for $\mathrm{P}_{x y l}$ is shown relative to the transcription start site [14]. 
cies, which are members of the GntR class of transcription factors [5,33]. As in C. crescentus, the hexuronateregulon in E. chrysanthemi includes the hexuronate isomerase pathway, extracellular enzymes for pectin breakdown, and inner membrane transporters (ExuT) [34,35]. Neither $C$. crescentus, its close relatives, or the Xanthomonas species encode KdgR-like genes. Instead, humR is embedded within the hexuronate metabolic gene cluster in the genomes of close C. crescentus relatives (C.segnis, C. henricii strain K31, Maricaulis maris, and Asticcacaulisexcentricus). Phenylobacterium zucineum has a modified cluster, with the HumR-regulated operon homologous to CC_1509-1508 (exuT) inserted between humR and $u x a C$. Based on the presence of HumR operators, the HumR regulon is largely conserved in these species, with the exception of CC_1446 and CC_3152, which are unique to $C$. crescentus. Xanthomonas campestris and $X$. axonopodis, which like Erwinia chrysanthemi are gamma-Proteobacterial phytopathogens [36], may also utilize a HumR-type regulatory system, as their genomes have a HumR homolog located immediately adjacent to $k d u I$ and $k d u D$, and possible operators located upstream of the $k d u I D$ operon and the $k d g K$ gene (not shown).

Genomic clustering of the genes for hexuronate metabolism (CC_1487 - CC_1496) undoubtedly has facilitated their co-regulation, and potential to be horizontally transferred as a functional unit. The breakdown of synteny outside of this gene cluster is consistent with the hypothesis that horizontal transfer introduced this pathway into the Caulobacter lineage. The inclusion of a simple transcription control system in the cluster was likely beneficial in that it ensured appropriate regulation of the pathway in a new host. Price et al. [37] found in E. coli that most "neighbor regulators"- transcription factors that are genetically adjacent to the genes they regulatewere likely acquired by horizontal gene transfer with their target genes. A similar pattern applies to other-LacItype repressors in the Caulobactergroup, since the hexuronate, glucose, and maltose-responsive repressors are clustered with genes they control in the $C$. crescentus genome, and XylR is clusted with the $x y l$ operon in the $C$. henricii K31 genome. Such clustering should help in elucidating the regulatory function of the many uncharacterized LacI homologs in the Caulobacter group.

\section{CONCLUSION}

Caulobacter crescentus encodes genes (CC_1487 to CC_1495) necessary for metabolism of galacturonate through the hexuronate isomerase pathway. A conserved $14 \mathrm{bp}$ operator upstream of three operons within this cluster binds HumR, a LacI-type repressor encoded by the CC_1489 gene. HumR represses these promoters in the absence of galacturonate, and releases the operator in its presence. Several additional genes likely involved in pectin degradation and hexuronate transport also appear to be part of the HumR regulon, which joins a growing roster of plant polysaccharide utilization systems controlled by LacI repressor homologs in C. crescentus.

\section{ACKNOWLEDGEMENTS}

This work was supported by U.S. National Science Foundation grant MCB-0818934 to CS, and by financial support for the BIOL $176 \mathrm{Re}$ combinant DNA Technology course from Santa Clara University. We gratefully acknowledge the help of numerous BIOL 176 students who contributed to this project through preparation of reagents and helpful discussions. We also thank Virginia Kalogeraki, Eduardo Abeliuk, Bo Zhou, and Harley McAdams for assistance with microarray experiments and discussion of data.

\section{REFERENCES}

[1] Mohnen, D. (2008) Pectin structure and biosynthesis. Current Opinion in Plant Biology, 11, 266-277. http://dx.doi.org/10.1016/j.pbi.2008.03.006

[2] Abbott, D.W. and Boraston, A.B. (2008) Structural biology of pectin degradation by Enterobacteriaceae. Microbiology and Molecular Biology Reviews, 72, 301-316. http://dx.doi.org/10.1128/MMBR.00038-07

[3] Richard, P. and Hilditch, S. (2009) D-galacturonic acid catabolism in microorganisms and its biotechnological relevance. Applied Microbiology and Biotechnology, 82, 597-604. http://dx.doi.org/10.1007/s00253-009-1870-6

[4] Yadav, S., Yadav, P., Yadav, D. and Yadav, K. (2009) Pectin lyase: A review. Process Biochemistry, 44, 1-10. http://dx.doi.org/10.1016/j.procbio.2008.09.012

[5] Hugouvieux-Cotte-Pattat, N. and Robert-Baudouy, J. (1987) Hexuronate catabolism in Erwinia chrysanthemi. Journal of Bacteriology, 169, 1223-1231.

[6] Entcheva-Dimitrov, P. and Spormann, A.M. (2004) Dynamics and control of biofilms of the oligotrophic bacterium Caulobacter crescentus. Journal of Bacteriology, 186, 8254-8266. http://dx.doi.org/10.1128/JB.186.24.8254-8266.2004

[7] Poindexter, J. (1964) Biological properties and classification of the Caulobacter group. Bacteriology Reviews, 28, 231-295.

[8] Nierman, W.C., Feldblyum, T.V., Laub, M.T., Paulsen, I.T., Nelson, K.E., Eisen, J., et al. (2001) Complete genome sequence of Caulobacter crescentus. Proceedings of the National Academy of Sciences of USA, 98, 4136-4141. http://dx.doi.org/10.1073/pnas.061029298

[9] Mannisto, M.K., Tiirola, M.A., Salkinoja-Salonen, M.S., Kulomaa, M.S. and Puhakka, J.A. (1999) Diversity of chlorophenol-degrading bacteria isolated from contaminated boreal groundwater. Archives of Microbiology, 171, 189197. http://dx.doi.org/10.1007/s002030050698

[10] Luo, Y., Xu, X., Ding, Z., Liu, Z., Zhang, B., Yan, Z., Sun, J., Hu, S. and Hu, X. (2008) Complete genome of Phenylobacterium zucineum, a novel facultative intracel- 
lular bacterium isolated from human erythroleukemia cell line K562. BioMed Central Genomics, 9, 386. http://dx.doi.org/10.1186/1471-2164-9-386

[11] Hottes, A.K., Meewan, M., Yang, D., Arana, N., Romero, P., McAdams, H.H. and Stephens, C. (2004) Transcriptional profiling of Caulobacter crescentus during growth on complex and minimal media. Journal of Bacteriology, 186, $1448-1461$. http://dx.doi.org/10.1128/JB.186.5.1448-1461.2004

[12] Stephens, C., Christen, B., Fuchs, T., Sundaram, V., Watanabe, K. and Jenal, U. (2007) Genetic analysis of a novel pathway for D-xylose metabolism in Caulobacter crescentus. Journal of Bacteriology, 189, 2181-2185. http://dx.doi.org/10.1128/JB.01438-06

[13] Stephens, C., Christen, B., Watanabe, K., Fuchs, T. and Jenal, U. (2008) Regulation of D-xylose metabolism in Caulobacter crescentus by a LacI-type repressor. Journal of Bacteriology, 189, 8828-8834. http://dx.doi.org/10.1128/JB.01342-07

[14] Meisenzahl, A.C., Shapiro, L. and Jenal, U. (1997) Isolation and characterization of a xylose-dependent promoter from Caulobacter crescentus. Journal of Bacteriology, 179, 592-600.

[15] Ely, B. (1991) Genetics of Caulobacter crescentus. Methods in Enzymology, 204, 372-384. http://dx.doi.org/10.1016/0076-6879(91)04019-K

[16] Bochner, B.R. (2003) New technologies to assess genotype-phenotype relationships. Nature Reviews Genetics, 4, 309-314. http://dx.doi.org/10.1038/nrg1046

[17] West, L., Yang, D. and Stephens, C. (2002) Use of the Caulobacter crescentus genome sequence to develop a method for systematic genetic mapping. Journal of Bacteriology, 184, 2155-2166. http://dx.doi.org/10.1128/JB.184.8.2155-2166.2002

[18] Zuker, M. (2003) Mfold web server for nucleic acid folding and hybridization prediction. Nucleic Acids Research, 31, 3406-3415. http://dx.doi.org/10.1093/nar/gkg595

[19] Stephens, C. and Shapiro, L. (1993) An unusual promoter controls cell-cycle regulation and dependence on DNA replication of the Caulobacter fliLM early flagellar operon. Molecular Microbiology, 9, 1169-1179. http://dx.doi.org/10.1111/j.1365-2958.1993.tb01246.x

[20] Hruz, T., Wyss, M., Docquier, M., Pfaffl, M., Masanetz, S., Borghi, L., et al. (2011) RefGenes: identification of reliable and condition specific reference genes for RTqPCR data normalization. BMC Genomics, 12, 156. http://dx.doi.org/10.1186/1471-2164-12-156

[21] Lohmiller, S., Hantke, K., Patzer, S.I. and Braun, V. (2008) TonB-dependent maltose transport by Caulobacter crescentus. Microbiology, 154, 1748-1754. http://dx.doi.org/10.1099/mic.0.2008/017350-0

[22] Livak, K.J. and Schmittgen, T.D. (2001) Analysis of relative gene expression data using real-time quantitative PCR and the $2[-\Delta \Delta \mathrm{C}[\mathrm{T}]]$ method]. Methods, 25, 402-408. http://dx.doi.org/10.1006/meth.2001.1262

[23] Williams, K.P., Sobral, B. and Dickerman, A. (2007) A robust species tree for the Alphaproteobacteria. Journal of Bacteriology, 189, 4578-4586.
http://dx.doi.org/10.1128/JB.00269-07

[24] Boer, H., Maaheimo, H., Koivula, A., Penttila, M. and Richard, P. (2009) Identification in Agrobacterium tumefaciens of the galacturonic acid dehydrogenase gene. Applied Microbiology and Biotechnology, 86, 901-909. http://dx.doi.org/10.1007/s00253-009-2333-9

[25] Riley, R.G. and Kolodziej, B.J. (1976) Pathway of glucose catabolism in Caulobacter crescentus. Microbios, 1, 219-226.

[26] Bailey, T., Bodén, M., Buske, F.A., Frith, M., Grant, C.E., Clementi, L., et al. (2009) MEME SUITE: Tools for motif discovery and searching. Nucleic Acids Research, 37, W202-W208. http://dx.doi.org/10.1093/nar/gkp335

[27] Fischer, M., Zhang, Q.Y., Hubbard, R.E. and Thomas, G.H. (2010) Caught in a TRAP: Substrate-binding proteins in secondary transport. Trends in Microbiology, 18, 471-478. http://dx.doi.org/10.1016/j.tim.2010.06.009

[28] Haseloff, B.J., Freeman, T.L., Valmeekam, V., Melkus, M.W., Oner, F., Valachovic, M.S. and San Francisco, M.J. (1998) The exuT gene of Erwinia chrysanthemi EC16: Nucleotide sequence, expression, localization, and relevance of the gene product. Molecular Plant-Microbe Interactions, 11, 270-276.

http://dx.doi.org/10.1094/MPMI.1998.11.4.270

[29] Collmer, A., Whalen, C., Beer, S. and Bateman, D. (1982) An exo-poly- $\alpha$-D-galacturonosidase implicated in the regulation of extracellular pectatelyase production in Erwiniachrystanthemi. Journal of Bacteriology, 149, 626-634.

[30] Swint-Kruse, L. and Matthews, K. (2009) Allostery in the LacI/GalR family: Variations on a theme. Current Opinions in Microbiology, 12, 129-137. http://dx.doi.org/10.1016/j.mib.2009.01.009

[31] Lin, J.-S. and Shaw, G.-C. (2007) Regulation of the kduID operon of Bacillus subtilis by the KdgR repressor and the ссрA gene: Identification of two KdgR-binding sites within the kdgR-kduI intergenic region. Microbiology, 153, 701-710. http://dx.doi.org/10.1099/mic.0.2006/002253-0

[32] Blanvillain, S., Meyer, D., Boulanger, A., Lautier, M., Guynet, C., Denanc, Ä.N., Vasse, J., Lauber, E. and Arlat, M. (2007) Plant carbohydrate scavenging through TonBdependent receptors: A feature shared by phytopathogenic and aquatic bacteria. PLoS One, 2, e224. http://dx.doi.org/10.1371/journal.pone.0000224

[33] Hugouvieux-Cotte-Pattat, N., Condemine, G., Nassar, W. and Reverchon, S. (1996) Regulation of pectinolysis in Erwinia chrysanthemi. Annual Review of Microbiology, 50, 213-257. http://dx.doi.org/10.1146/annurev.micro.50.1.213

[34] Rodionov, D.A., Gelfand, M.S. and Hugouvieux-CottePattat, N. (2004) Comparative genomics of the KdgR regulon in Erwinia chrysanthemi 3937 and other gammaproteobacteria. Microbiology, 150, 3571-3590. http://dx.doi.org/10.1099/mic.0.27041-0

[35] Rodionov, D.A., Mironov, A.A., Rakhmaninova, A.B. and Gelfand, M.S. (2000) Transcriptional regulation of transport and utilization systems for hexuronides, hexuronates and hexonates in gamma purple bacteria. Molecular Microbiology, 38, 673-683. http://dx.doi.org/10.1046/j.1365-2958.2000.02115.x 
[36] Da Silva, A.C., Ferro, J.A., Reinach, F.C., Farah, C.S., Furlan, L.R., Quaggio, R.B., et al. (2002) Comparison of the genomes of two Xanthomonas pathogens with differing host specificities. Nature, 417, 459-463.

http://dx.doi.org/10.1038/417459a
[37] Price, M.N., Dehal, P. and Arkin, A. (2008) Horizontal gene transfer and the evolution of transcriptional regulation in Escherichia coli. Genome Biology, 9, R4. http://dx.doi.org/10.1186/gb-2008-9-1-r4 